\title{
Structural Damage Detection by Using Single Natural Frequency and the Corresponding Mode Shape
}

\author{
Bo Zhao, Zili Xu, Xuanen Kan, Jize Zhong, and Tian Guo \\ State Key Laboratory for Strength and Vibration of Mechanical Structures, Xian Jiaotong University, Xian 710049, China \\ Correspondence should be addressed to Zili Xu; zlxu@mail.xjtu.edu.cn
}

Received 17 August 2015; Revised 29 October 2015; Accepted 16 November 2015

Academic Editor: Juan P. Amezquita-Sanchez

Copyright ( 2016 Bo Zhao et al. This is an open access article distributed under the Creative Commons Attribution License, which permits unrestricted use, distribution, and reproduction in any medium, provided the original work is properly cited.

\begin{abstract}
Damage can be identified using generalized flexibility matrix based methods, by using the first natural frequency and the corresponding mode shape. However, the first mode is not always appropriate to be used in damage detection. The contact interface of rod-fastened-rotor may be partially separated under bending moment which decreases the flexural stiffness of the rotor. The bending moment on the interface varies as rotating speed changes, so that the first- and second-modal parameters obtained are corresponding to different damage scenarios. In this paper, a structural damage detection method requiring single nonfirst mode is proposed. Firstly, the system is updated via restricting the first few mode shapes. The mass matrix, stiffness matrix, and modal parameters of the updated system are derived. Then, the generalized flexibility matrix of the updated system is obtained, and its changes and sensitivity to damage are derived. The changes and sensitivity are used to calculate the location and severity of damage. Finally, this method is tested through numerical means on a cantilever beam and a rod-fastened-rotor with different damage scenarios when only the second mode is available. The results indicate that the proposed method can effectively identify single, double, and multiple damage using single nonfirst mode.
\end{abstract}

\section{Introduction}

Damage in a structure produces variations in its geometric and physical properties, which can result in changes in its natural frequencies and mode shapes. In the last years, several researchers have developed many damage detection methods based on dynamic parameters. Fan and Qiao [1] and Jassim et al. [2] presented comprehensive reviews on modal parameters-based damaged identification methods. The most commonly used methods of damage detection use changes of natural frequencies and mode shape directly. Messina et al. [3] proposed a correlation coefficient termed the Multiple Damage Location Assurance Criterion (MDLAC) by introducing two methods for estimating the location and size of defects in a structure. Kim and Stubbs [4] proposed a single damage indicator (SDI) method to locate and quantify a single crack in slender structures by using changes in a few natural frequencies. Xu et al. [5] proposed an iterative algorithm to identify the locations and extent of damage in beams only using the changes in their first several natural frequencies. However, the natural frequency-based methods are often ill-posed even without noise. Shi et al. [6] extended the Multiple Damage Location Assurance Criterion (MDLAC) by using incomplete mode shapes instead of natural frequencies. Pawar et al. [7] proposed a method of damage detection using Fourier analysis of mode shapes and neural networks, which is limited to detecting damage of beams with clamped-clamped boundary condition. Another important class of damage detection methods is based on flexibility matrix change between damaged and undamaged structures. Pandey and Biswas [8] first proposed the method based on change in flexibility matrix to detect structural damage. Yang and Liu [9] made use of the eigenparameter decomposition of structural flexibility matrix change and approached the location and severity of damage in a decoupled manner. Bernal and Gunes [10] use the flexibility proportional matrix method to quantify damage without the use of a model. Tomaszewska [11] investigated the effect of statistical errors on damage detection based on structural flexibility matrix and mode shape curvature. Li et al. [12] used the generalized flexibility instead of original flexibility matrix to detect structural damage, which can significantly reduce the effect 
of truncating higher-order modal parameters. Masoumi et al. [13] proposed a new objective function formed by using generalized flexibility matrix. Then, imperialist competitive algorithm was used in damage identification. Yan and Ren [14] derived a closed form of the sensitivity of flexibility based on the algebraic eigensensitivity method. Montazer and Seyedpoor [15] introduced a new flexibility based damage index for damage detection of truss structures.

Although the generalized flexibility matrix based damage detection approach can precisely detect the location and severity of damage by using only the first natural frequency and the corresponding mode shape, there are still many limitations in these methods. One limitation lies in the damage detection of rod-fastened-rotor of heavy duty gas turbine. The flexural stiffness of the interface decreases when some zones of the contact interface are separated with bending moment on the rotor [16]. Flexural stiffness of interface in a rod-fastened-rotor induced by bending moment is different in first and second critical speed, because bending moment distribution varies as rotating speed changes. Therefore, only the second-modal parameters are available for the damage detection of rod-fastened-rotor in the second critical speed.

In this paper, a structural damage detection method based on changes in the flexibility matrix only using single natural frequency and the corresponding mode shape is presented. Firstly, restricted by the first several mode shapes, the system is updated. The flexibility matrix of updated system can be obtained by using non-first-modal parameters of original system. Then, sensitivity of flexibility of the updated system to damage is derived. Taking advantage of generalized flexibility matrix, which can considerably reduce the error caused by truncating higher-order modal parameters, the location and severity of the damage are calculated. Finally, two numerical examples for a cantilever beam and a rod-fastened-rotor are used to illustrate the effectiveness of the proposed method, when only the second natural frequency and the corresponding mode shape are available.

\section{Structural Damage Detection Method}

2.1. Structural System Updating Method. The differential equation governing the free vibration of a linear, undamped structural system can be expressed as

$$
\mathbf{M} \ddot{\mathbf{x}}+\mathbf{K x}=0,
$$

where $\mathbf{M}$ is the global mass matrix, $\mathbf{K}$ is the global stiffness matrix, and $\mathbf{x}$ is the displacement vector. When the degree of freedom for the system is $n$, the eigenvalue problem can be written in the form

$$
\mathbf{K} \Phi_{i}=\lambda_{i} \mathbf{M} \Phi_{i}, \quad i=1,2, \ldots, n,
$$

where $\lambda_{i}$ and $\boldsymbol{\Phi}_{i}$ are the $i$ th eigenvalue and eigenvector, respectively. Restricting the system by the first $r$ mode shapes,

$$
\boldsymbol{\Phi}_{i}^{T} \mathbf{M x}=0, \quad i=1,2, \ldots, r .
$$

Mode shape matrix, mass matrix, and displacement vector can be partitioned as

$$
\begin{aligned}
& \boldsymbol{\Psi}=\left[\begin{array}{ll}
\boldsymbol{\Psi}_{r r} & \boldsymbol{\Psi}_{r s} \\
\boldsymbol{\Psi}_{s r} & \boldsymbol{\Psi}_{s s}
\end{array}\right], \\
& \mathbf{M}=\left[\begin{array}{ll}
\mathbf{M}_{r r} & \mathbf{M}_{r s} \\
\mathbf{M}_{s r} & \mathbf{M}_{s s}
\end{array}\right], \\
& \mathbf{x}=\left[\begin{array}{l}
\mathbf{x}_{r} \\
\mathbf{x}_{s}
\end{array}\right],
\end{aligned}
$$

where the $i$ th column of $\boldsymbol{\Psi}$ is the $i$ th eigenvector $\boldsymbol{\Phi}_{i}$. Substituting (4) into (3) yields

$$
\left[\begin{array}{ll}
\boldsymbol{\Psi}_{r r}^{T} & \boldsymbol{\Psi}_{s r}^{T}
\end{array}\right]\left[\begin{array}{ll}
\mathbf{M}_{r r} & \mathbf{M}_{r s} \\
\mathbf{M}_{s r} & \mathbf{M}_{s s}
\end{array}\right]\left[\begin{array}{l}
\mathbf{x}_{r} \\
\mathbf{x}_{s}
\end{array}\right]=0 .
$$

Expending (5) yields $\mathbf{x}_{r}=-\left(\boldsymbol{\Psi}_{r r} \mathbf{M}_{r r}+\boldsymbol{\Psi}_{r s} \mathbf{M}_{s r}\right)^{-1}\left(\boldsymbol{\Psi}_{r r} \mathbf{M}_{r s}+\right.$ $\left.\Psi_{r s} \mathbf{M}_{s s}\right) \mathbf{x}_{s}$; then the relationship between $\mathbf{x}_{s}$ and $\mathbf{x}$ is

$$
\mathbf{x}=\mathbf{D} \mathbf{x}_{s}
$$

where $\mathbf{D}=\left[\begin{array}{c}\mathrm{TR} \\ \mathbf{I}\end{array}\right]$, in which $\mathbf{T R}=-\left(\boldsymbol{\Psi}_{r r} \mathbf{M}_{r r}+\right.$ $\left.\boldsymbol{\Psi}_{r s} \mathbf{M}_{s r}\right)^{-1}\left(\boldsymbol{\Psi}_{r r} \mathbf{M}_{r s}+\boldsymbol{\Psi}_{r s} \mathbf{M}_{s s}\right) \mathbf{x}_{s}$. Substituting (6) into original free vibration differential equation (1) yields

$$
\mathbf{M D} \ddot{\mathbf{x}}_{s}+\mathbf{K D x}_{s}=0
$$

Left-multiplying (7) by $\mathbf{D}^{T}$ yields the updated free vibration differential equation

$$
\mathbf{M}^{u} \ddot{\mathbf{x}}_{s}+\mathbf{K}^{u} \mathbf{x}_{s}=0
$$

The mass and stiffness matrix of the updated system can be obtained by

$$
\begin{aligned}
\mathbf{M}^{u} & =\mathbf{D}^{T} \mathbf{M D}, \\
\mathbf{K}^{u} & =\mathbf{D}^{T} \mathbf{K D} .
\end{aligned}
$$

The relationship between the updated and the original modal parameters can be described by

$$
\begin{aligned}
& \lambda_{i}^{u}=\lambda_{i+r}, \\
& \boldsymbol{\Phi}_{i}^{u}=\mathbf{D}^{-1} \boldsymbol{\Phi}_{i+r}, \\
& \quad i=1,2, \ldots, n-r,
\end{aligned}
$$

where $\lambda_{i}^{u}$ and $\Phi_{i}^{u}$ are the $i$ th eigenvalue and eigenvector of the updated system, respectively. $\mathbf{D}^{-1}$ is a generalized inverse of $\mathbf{D}$, because $\mathbf{D}$ is not a square matrix. Thus, a new $n-r$ dimension system based on the original $n$ dimension system is established.

The complete mode shapes are difficult to obtain, particularly when a limited number of sensors are available. However, incomplete mode shape data can be expanded to 
complete mode shapes by mode shape expansion technique. The expansion method in [17] is

$$
\boldsymbol{\Phi}_{j}=\left[\begin{array}{c}
\mathbf{I} \\
-\left(\mathbf{K}_{s s}-\lambda_{j} \mathbf{M}_{s s}\right)^{-1}\left(\mathbf{K}_{s m}-\lambda_{j} \mathbf{M}_{s m}\right)
\end{array}\right] \boldsymbol{\Phi}_{m j},
$$

where $\boldsymbol{\Phi}_{m j}$ is measured degrees of mode shape $\boldsymbol{\Phi}_{j}$ and $\mathbf{K}_{s s}$, $\mathbf{K}_{s m}$ and $\mathbf{M}_{s s}, \mathbf{M}_{s m}$ are submatrix of global stiffness and mass matrix, respectively.

2.2. Structural Damage Detection Based on Generalized Flexibility Matrix Method. In this method, only the decrease in structure stiffness due to damage is considered. Changes in mass property are ignored. The damage parameters are denoted by $d_{i}$, which stands for damage extent of the $i$ th element. The decrease of global stiffness matrix $\Delta \mathbf{K}$ can be expressed as a sum of each elemental stiffness matrix multiplied by damage parameters [9]; that is,

$$
\Delta \mathbf{K}=\mathbf{K}_{a}-\mathbf{K}_{d}=\sum_{i=1}^{e} d_{i} \mathbf{K}_{a i}
$$

where $\mathbf{K}_{a}$ is the global stiffness matrix of undamaged structure, $\mathbf{K}_{d}$ is the global stiffness matrix of damaged structure, and $\mathbf{K}_{a i}$ is the $i$ th elemental stiffness matrix positioned within the global matrix for undamaged structure, and $e$ is the number of elements. If the $i$ th element is undamaged, the value of $d_{i}$ is zero. The value of $d_{i}$ is a nonnegative number less than one. Differentiating (12) with respect to $d_{i}$ leads to

$$
\frac{\partial \mathbf{K}_{d}}{\partial d_{i}}=-\mathbf{K}_{a i} .
$$

According to the definition of flexibility and stiffness matrix, they satisfy the following relationship:

$$
\mathbf{F}_{d}^{u} \mathbf{K}_{d}^{u}=\mathbf{I}
$$

where $\mathbf{F}_{d}^{u}$ is the flexibility matrix of updated system for the damaged structure, $\mathbf{K}_{d}^{u}$ is the stiffness matrix of updated system for the damaged structure, and $\mathbf{I}$ is the identity matrix. Differentiating (14) with respect to $d_{i}$ leads to

$$
\frac{\partial \mathbf{F}_{d}^{u}}{\partial d_{i}} \mathbf{K}_{d}^{u}=-\mathbf{F}_{d}^{u} \frac{\partial \mathbf{K}_{d}^{u}}{\partial d_{i}} .
$$

Postmultiplying (15) by $\mathbf{F}_{d}^{u}$ yields

$$
\frac{\partial \mathbf{F}_{d}^{u}}{\partial d_{i}}=-\mathbf{F}_{d}^{u} \frac{\partial \mathbf{K}_{d}^{u}}{\partial d_{i}} \mathbf{F}_{d}^{u}
$$

As the damage is a small amount, $\mathbf{F}_{d}^{u} \approx \mathbf{F}_{a}^{u}$ is satisfied. Substituting (9) and (13) into (16), the sensitivity of flexibility matrix to damage for the new system can be derived as

$$
\begin{aligned}
\frac{\partial \mathbf{F}^{u}}{\partial d_{i}} & \approx-\mathbf{F}^{u} \frac{\partial \mathbf{K}^{u}}{\partial d_{i}} \mathbf{F}^{u} \\
& =\left(\mathbf{D}^{T} \mathbf{K D}\right)^{-1} \mathbf{D}^{T} \mathbf{K}_{a i} \mathbf{D}\left(\mathbf{D}^{T} \mathbf{K D}\right)^{-1} .
\end{aligned}
$$

In order to reduce the error result from truncating higherorder modes, generalized flexibility matrix $\mathbf{f}^{u}=\mathbf{F}^{u}\left(\mathbf{M}^{u} \mathbf{F}^{u}\right)^{l}$ is used [12]. In this research, $l=2$ is adopted. The generalized flexibility matrix for the updated system can be written as

$$
\mathbf{f}^{u}=\mathbf{F}^{u} \mathbf{M}^{u} \mathbf{F}^{u} \mathbf{M}^{u} \mathbf{F}^{u} .
$$

Differentiating (18) with respect to $d_{i}$ leads to

$$
\begin{aligned}
\frac{\partial \mathbf{f}^{u}}{\partial d_{i}}= & \frac{\partial \mathbf{F}^{u}}{\partial d_{i}} \mathbf{M}^{u} \mathbf{F}^{u} \mathbf{M}^{u} \mathbf{F}^{u}+\mathbf{F}^{u} \mathbf{M}^{u} \frac{\partial \mathbf{F}^{u}}{\partial d_{i}} \mathbf{M}^{u} \mathbf{F}^{u} \\
& +\mathbf{F}^{u} \mathbf{M}^{u} \mathbf{F}^{u} \mathbf{M}^{u} \frac{\partial \mathbf{F}^{u}}{\partial d_{i}} .
\end{aligned}
$$

Combining (17) and (19), the sensitivity of generalized flexibility matrix to damage can be obtained. Making use of Taylor's series expansion, change in generalized flexibility matrix can be described as

$$
\Delta \mathbf{f}^{u} \approx \sum_{i=1}^{e} \frac{\partial \mathbf{f}^{u}}{\partial d_{i}} d_{i}
$$

The generalized flexibility matrix for the updated system can also be approximately determined by using its first frequency $\lambda_{1}^{u}$ and the corresponding mode $\Phi_{1}^{u}$, which can be acquired by the $r+1$ th frequency $\lambda_{r+1}$ and the corresponding mode $\boldsymbol{\Phi}_{r+1}$ of original system, respectively. Then change in generalized flexibility matrix can be described as

$$
\begin{aligned}
\Delta \mathbf{f}^{u} \approx & \frac{1}{\lambda_{d, r+1}^{3}}\left(\mathbf{D}^{-1} \boldsymbol{\Phi}_{d, r+1}\right)\left(\mathbf{D}^{-1} \boldsymbol{\Phi}_{d, r+1}\right)^{T} \\
& -\frac{1}{\lambda_{u, r+1}^{3}}\left(\mathbf{D}^{-1} \boldsymbol{\Phi}_{u, r+1}\right)\left(\mathbf{D}^{-1} \boldsymbol{\Phi}_{u, r+1}\right)^{T},
\end{aligned}
$$

where $\lambda_{d, r+1}$ and $\Phi_{d, r+1}$ are the $r+1$ th frequency and mode shape of the damaged structure and $\lambda_{a, r+1}$ and $\Phi_{a, r+1}$ are the $r+1$ th frequency and mode shape of the undamaged structure, respectively. When first $r$ modal parameters are unavailable, damage parameters can be acquired by manipulating (20) and (21) into a system of linear equations, which can be solved by using the least squares method.

\section{Numerical Examples}

In order to verify the effectiveness of the proposed method, two numerical examples are considered. The first numerical example is a cantilever beam, and the second one is a rodfastened-rotor considering partial separation of interface.

3.1. Forty-Five-Element Cantilevered Beam. A two-dimensional cantilever beam with a rectangular section, as shown in Figure 1, is taken as a case study to verify the effectiveness of the proposed method. The basic parameters of material and geometrics are as follows: elastic modulus $E=2.1 \mathrm{GPa}$, density $\rho=7800 \mathrm{~kg} / \mathrm{m}^{3}$, length $l=0.45 \mathrm{~m}$, cross section area $A=1.6129 \times 10^{-4} \mathrm{~m}^{2}$, and the moment of inertia $I=$ $5.42 \times 10^{-8} \mathrm{~m}^{4}$. The total number of elements and degrees 


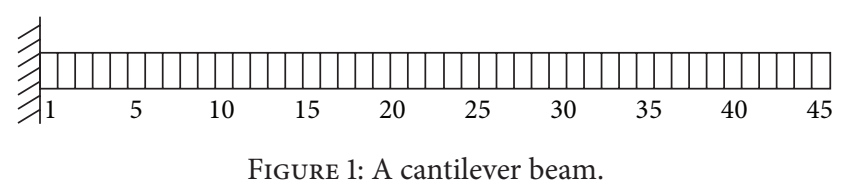

of freedom are 45 and 90, respectively. The length of each element is $0.01 \mathrm{~m}$. Two damage cases are presented here: case 1: element 28 is damaged with stiffness losses of $10 \%$; case 2 : elements 18 and 36 are damaged simultaneously with stiffness losses of $14 \%$ and $6 \%$, respectively.

When the first mode is unavailable, location and severity of damage can be obtained by using the second natural frequency and the corresponding mode shape with the proposed method in this paper. Parameter $r$ is the number of unavailable modes. Damage parameters can be acquired by solving (20) and (21) with $r=1$.

The results are also compared with the results obtained by using the method in [6], which is an extension of the Multiple Damage Location Assurance Criterion (MDLAC). Making use of the mode shape directly, the damage sites can be approximately localized as those sites with the large MDLAC values. The MDLAC value in [6] is

\section{$\operatorname{MDLAC}(\delta \mathbf{A})$}

$$
=\frac{\left|\Delta \boldsymbol{\Phi}^{T} \cdot \delta \boldsymbol{\Phi}(\delta \mathbf{A})\right|^{2}}{\left(\Delta \boldsymbol{\Phi}^{T} \cdot \Delta \boldsymbol{\Phi}\right) \cdot\left(\delta \boldsymbol{\Phi}(\delta \mathbf{A})^{T} \cdot \delta \boldsymbol{\Phi}(\delta \mathbf{A})\right)} .
$$

Figure 2 shows the results calculated by the methods proposed in this paper and [6] for damage case 1, which represents the case of single damage. The vertical axis of Figures 2(a) and 2(b) is absolute damage extent and normalized MDLAC value by using the methods proposed in this paper and [6], respectively. Results less than zero are ignored, because each $a_{i}$ is assumed to be a nonnegative number. Damaged element can be located accurately by both methods, while damage extent can also be detected by the proposed method in this paper.

Similarly, Figure 3 displays the results calculated by the proposed methods in this paper and the method in [6] for damage case 2 (double damage). While the method in [6] that directly uses mode shape fails to detect the damage in element 36 only using the second mode shape, the method proposed in this paper predicts the location of the damage successfully. The damage extent detected is 0.163 and 0.064 for elements 18 and 36 , respectively.

To consider effect of the measured noise of frequency and mode shape on accuracy of the proposed method, $1 \%$ and $5 \%$ random noise are added in the frequency and mode shape for damage detection, respectively $[6,18]$. Figure 4 shows the results calculated by the proposed method in this paper for damage cases 1 and 2 . The results show that accurate locations can also be estimated considering effect of measured noise.

To investigate effect of using incomplete mode shapes on accuracy of the proposed method, 80 and 60 nodal displacements of the complete second mode shape are used to detect the damage. The noise effect is also considered
TABLE 1: Location and extent of multiple damage of rod-fastenedrotor.

\begin{tabular}{lcccc}
\hline Element number & 10 & 11 & 12 & 13 \\
\hline Damage extent & $5.2 \%$ & $16.2 \%$ & $11.9 \%$ & $2.2 \%$ \\
\hline
\end{tabular}

at the same time. Figure 5 shows the results calculated by the method proposed in this paper for damage cases 1 and 2 when 80 nodal displacements of the second mode shape are available. Figure 6 displays the results calculated by the method presented in this paper for damage cases 1 and 2 when only 60 nodal displacements of the second mode shape are available.

3.2. Simplified Rod-Fastened-Rotor. The rod-fastened-rotors are commonly used in heavy duty gas turbines and aero engines. As shown in Figure 7, the rod-fastened-rotor is comprised of a battery of discs clamped together by tie rods. There is contact interface between discs of the rod-fastenedrotor. Some zones of the contact interface are separated and the flexural stiffness of the rod-fastened-rotor decreases when bending moment exceeds critical value [16].

Detection of partial separation of contact interface for a rod-fastened-rotor in heavy duty gas turbine is investigated in this paper. The beam elements are used to model the rodfastened-rotor. The total number of elements is 37 , as shown in Figure 7. The unbalance response and distribution of flexural moment are calculated by using the common commercial finite element software ANSYS. Figure 8 shows the flexural moment on each element at the second critical speed. According to the relationship between bending moment and flexural stiffness in [19], damage ratio of each element is shown in Table 1.

The location and extent of damage are detected with the method proposed in this paper and the result is shown in Figure 9. The damage extent of damaged elements 10, 11, 12 , and 13 detected by our method is $5.9 \%, 24 \%, 14 \%$, and $0.7 \%$, respectively. Although several undamaged elements are detected to be damaged with minor extent, the result can also help us to find the location of the damage.

From the results obtained above, the proposed method can effectively identify single, double, and multiple damage without the first frequency and the corresponding mode shape for numerical examples.

\section{Conclusions}

The first-modal parameter of system plays the most important role in its flexibility matrix; the original flexibility method cannot be used to detect damage when the firstmodal parameter is unavailable. A damage detection method based on flexibility change by using single nonfirst mode is presented. The system is updated via restricting the first few mode shapes. The mass matrix, stiffness matrix, and modal parameters of the updated system are obtained. Then sensitivity of the new flexibility matrix to damage is derived. The damage extent of each element can be calculated by solving a linear equation. This method is tested through 


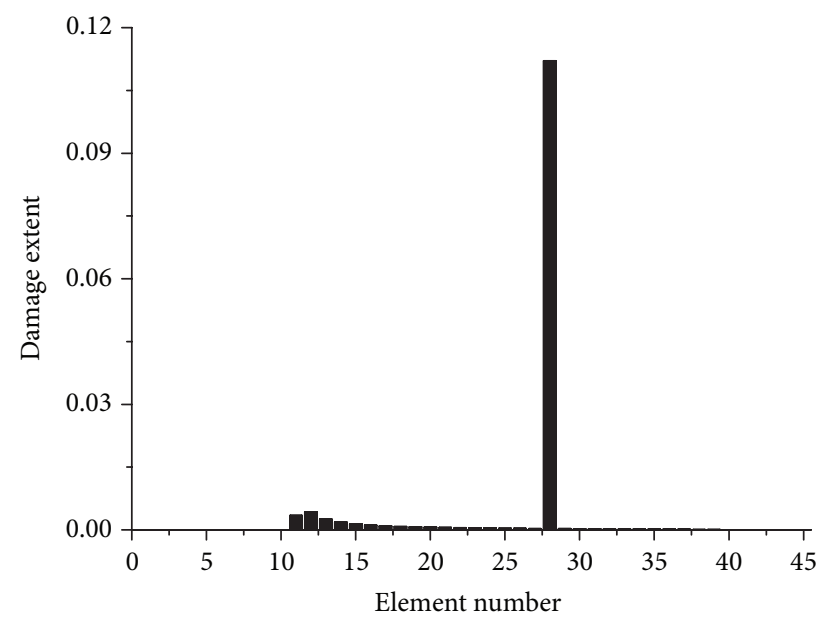

(a)

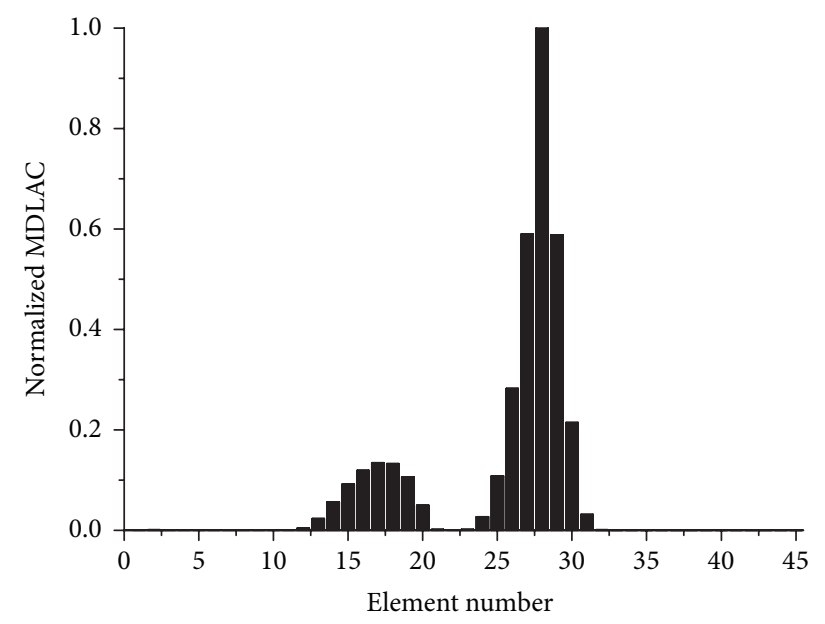

(b)

FIGURE 2: Damage detection by only using the second-modal parameters for damage case 1: (a) and (b) are the results by using the proposed method in this paper and the method in [6], respectively.

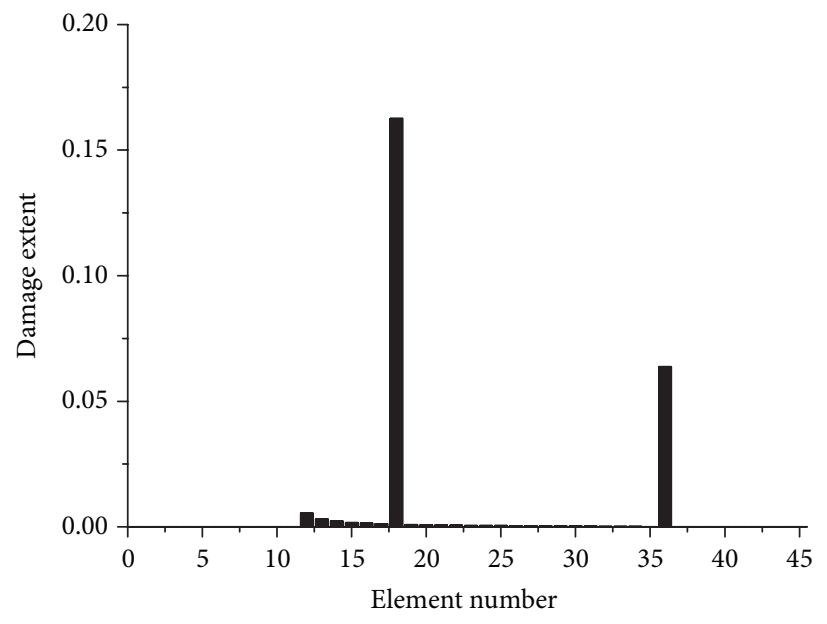

(a)

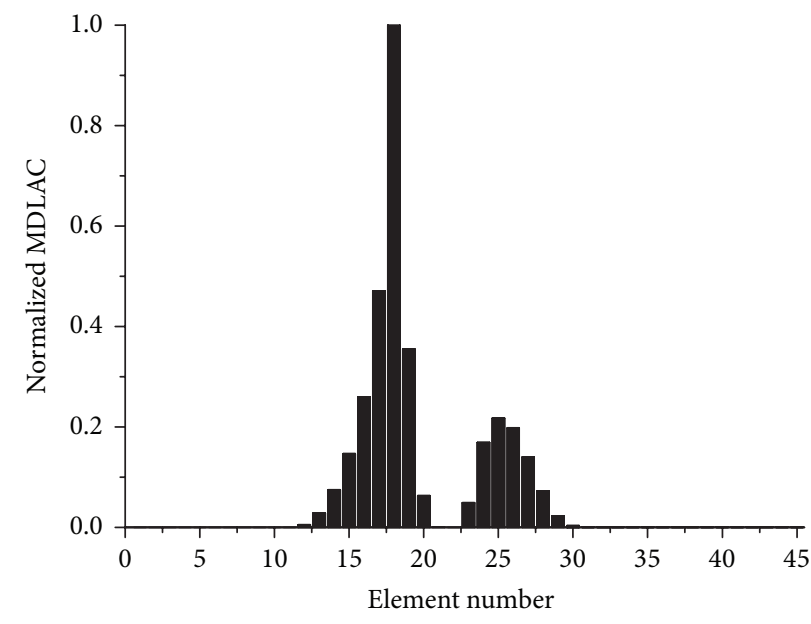

(b)

FiguRE 3: Damage detection by only using the second-modal parameters for damage case 2: (a) and (b) are the results by using the proposed method in this paper and the method in [6], respectively.

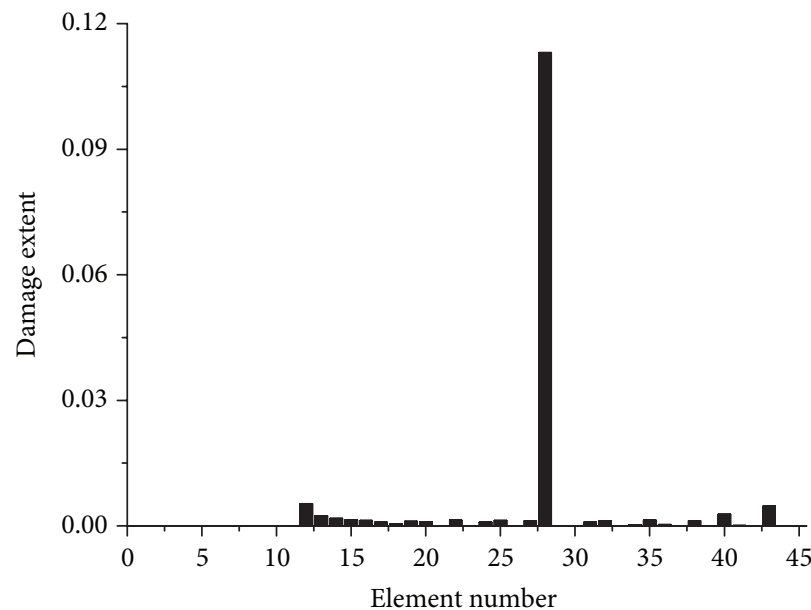

(a)

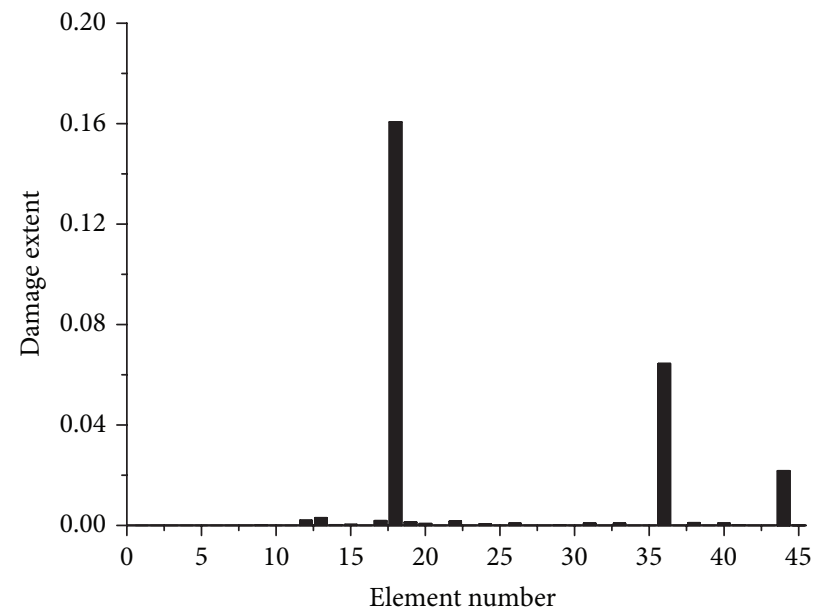

(b)

Figure 4: Damage detection by only using the second-modal parameters for damage (a) case 1 and (b) case 2, when $1 \%$ and $5 \%$ random noise are added in the frequencies and mode shapes, respectively. 


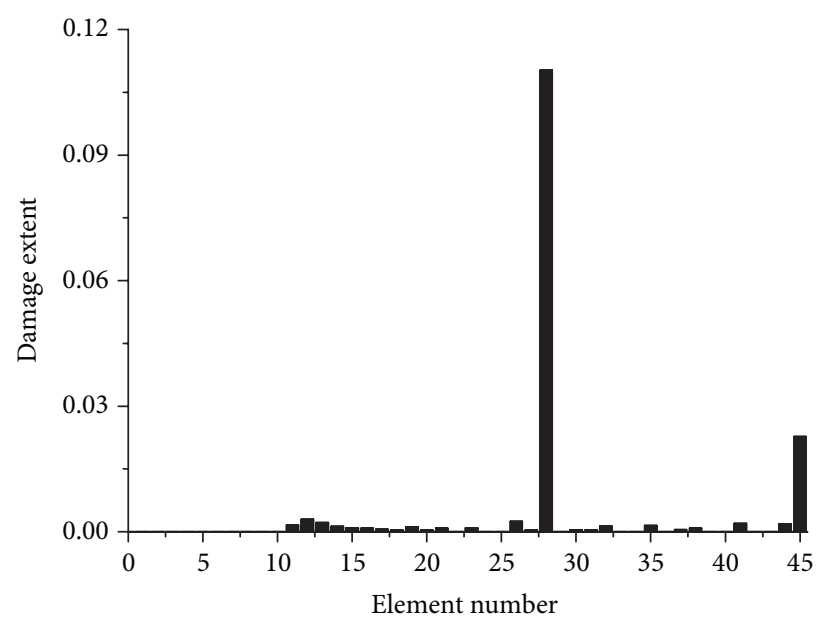

(a)

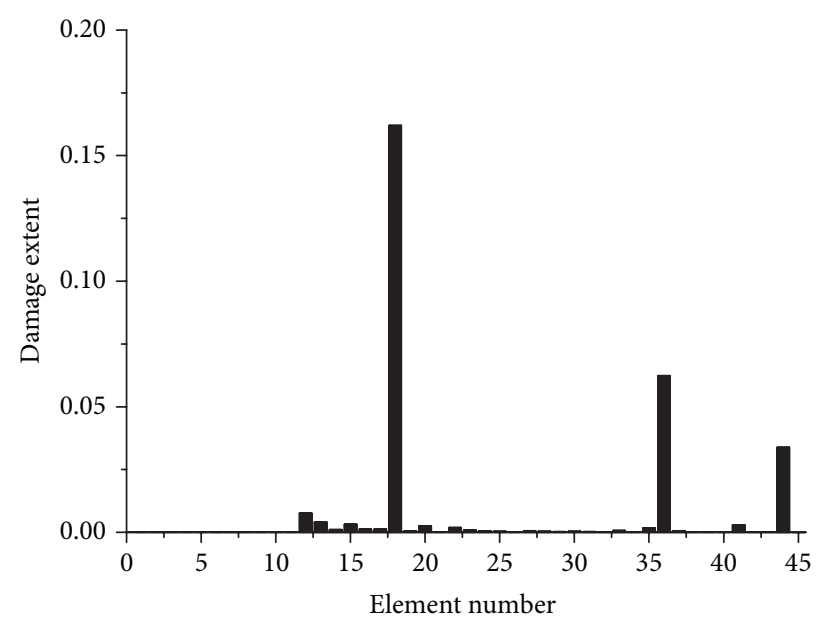

(b)

FIGURE 5: Damage detection by using the second natural frequency and 80 nodal displacements of the corresponding mode shape: (a) case 1; (b) case 2 .

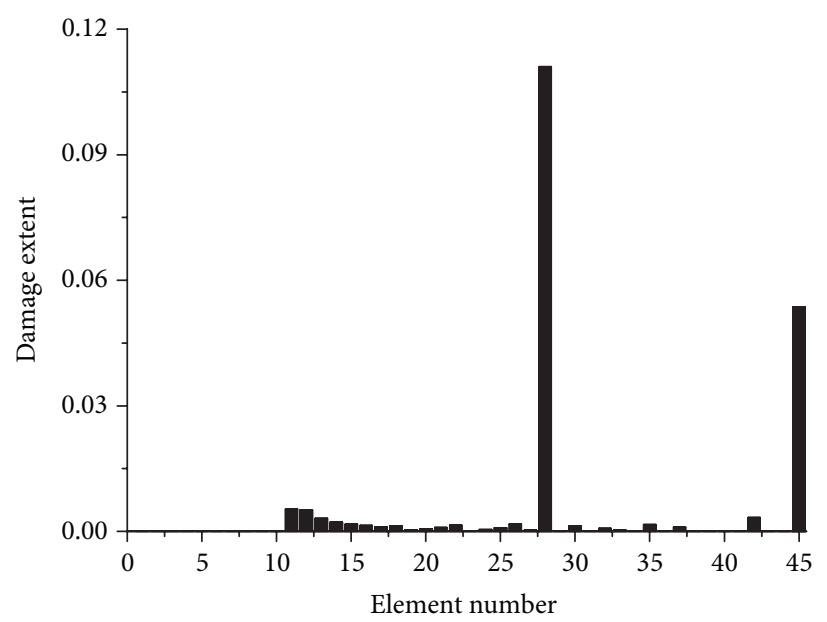

(a)

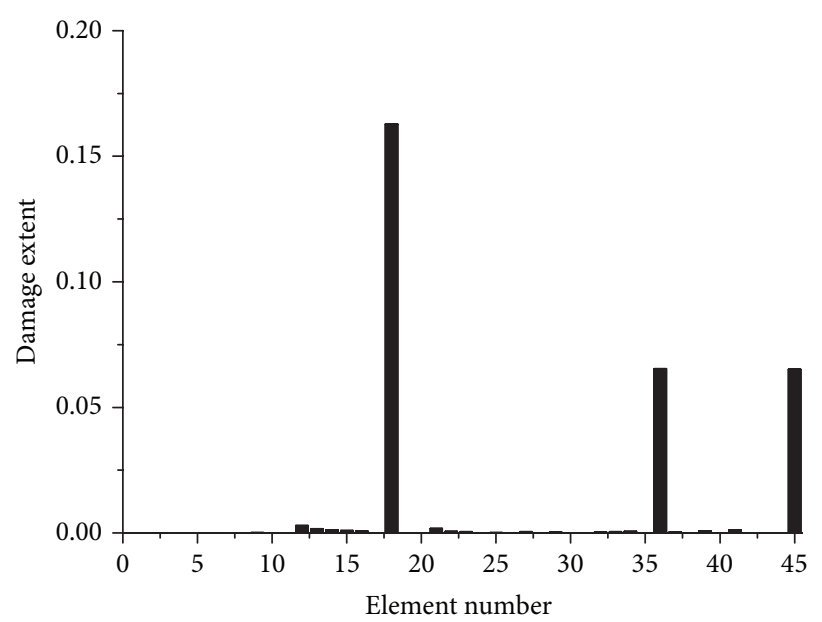

(b)

FiguRE 6: Damage detection by using the second natural frequency and 60 nodal displacements of the corresponding mode shape: (a) case 1; (b) case 2.

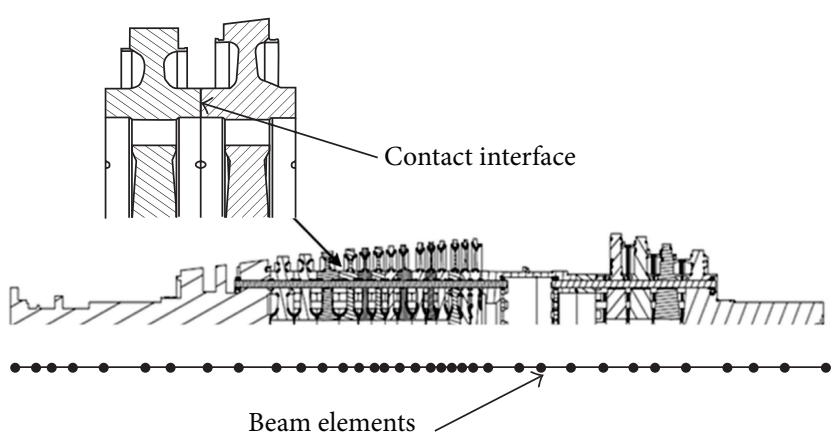

FIGURE 7: Schematic diagram of heavy duty gas turbine and node dividing. 


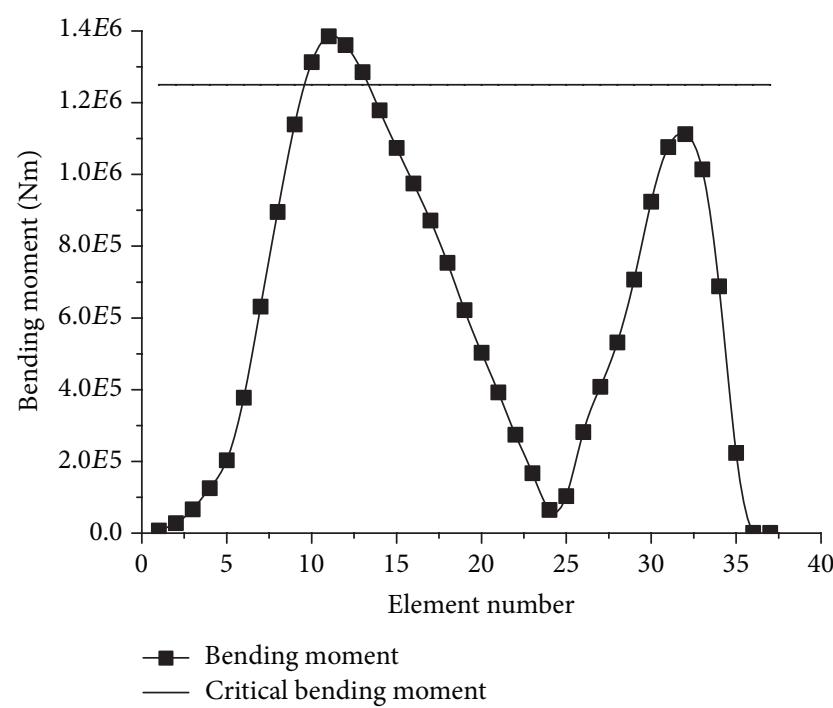

Figure 8: Bending moment on each interface of the rod-fastenedrotor at the second critical speed.

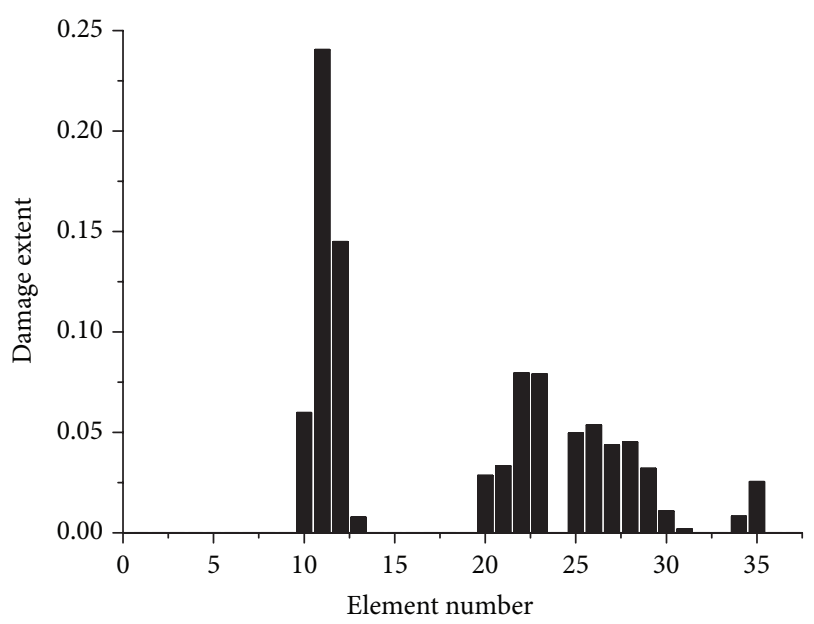

FIGURE 9: Damage detection by only using the second-modal parameter of the rod-fastened-rotor.

numerical means on a cantilever beam and a rod-fastenedrotor with different damage scenarios, when only the second natural frequency and mode shape are available. The results of the numerical examples indicate that the proposed method can effectively identify single, double, and multiple damage.

\section{Conflict of Interests}

The authors declare that there is no conflict of interests regarding the publication of this paper.

\section{Acknowledgments}

This work was supported by the Natural Science Foundation of China (no. 51275385) and Major State Basic Research Development Program of China (no. 2011CB706505).

\section{References}

[1] W. Fan and P. Z. Qiao, "Vibration-based damage identification methods: a review and comparative study," Structural Health Monitoring, vol. 10, no. 1, pp. 83-111, 2011.

[2] Z. A. Jassim, N. N. Ali, F. Mustapha, and N. A. Abdul Jalil, "A review on the vibration analysis for a damage occurrence of a cantilever beam," Engineering Failure Analysis, vol. 31, pp. 442461, 2013.

[3] A. Messina, E. J. Williams, and T. Contursi, "Structural damage detection by a sensitivity and statistical-based method," Journal of Sound and Vibration, vol. 216, no. 5, pp. 791-808, 1998.

[4] J.-T. Kim and N. Stubbs, "Crack detection in beam-type structures using frequency data," Journal of Sound and Vibration, vol. 259, no. 1, pp. 145-160, 2003.

[5] G. Y. Xu, W. D. Zhu, and B. H. Emory, "Experimental and numerical investigation of structural damage detection using changes in natural frequencies," Journal of Vibration and Acoustics, vol. 129, no. 6, pp. 686-700, 2007.

[6] Z. Y. Shi, S. S. Law, and L. M. Zhang, "Damage localization by directly using incomplete mode shapes," Journal of Engineering Mechanics, vol. 126, no. 6, pp. 656-660, 2000.

[7] P. M. Pawar, K. Venkatesulu Reddy, and R. Ganguli, "Damage detection in beams using spatial fourier analysis and neural networks," Journal of Intelligent Material Systems and Structures, vol. 18, no. 4, pp. 347-359, 2007.

[8] A. K. Pandey and M. Biswas, "Damage detection in structures using changes in flexibility," Journal of Sound and Vibration, vol. 169, no. 1, pp. 3-17, 1994.

[9] Q. W. Yang and J. K. Liu, "Damage identification by the eigenparameter decomposition of structural flexibility change," International Journal for Numerical Methods in Engineering, vol. 78, no. 4, pp. 444-459, 2009.

[10] D. Bernal and B. Gunes, "Flexibility based approach for damage characterization: benchmark application," Journal of Engineering Mechanics, vol. 130, no. 1, pp. 61-70, 2004.

[11] A. Tomaszewska, "Influence of statistical errors on damage detection based on structural flexibility and mode shape curvature," Computers \& Structures, vol. 88, no. 3-4, pp. 154-164, 2010.

[12] J. Li, B. Wu, Q. C. Zeng, and C. W. Lim, "A generalized flexibility matrix based approach for structural damage detection," Journal of Sound and Vibration, vol. 329, no. 22, pp. 4583-4587, 2010.

[13] M. Masoumi, E. Jamshidi, and M. Bamdad, "Application of generalized flexibility matrix in damage identification using Imperialist Competitive Algorithm," KSCE Journal of Civil Engineering, vol. 19, no. 4, pp. 994-1001, 2015.

[14] W.-J. Yan and W.-X. Ren, "Closed-form modal flexibility sensitivity and its application to structural damage detection without modal truncation error," Journal of Vibration and Control, vol. 20, no. 12, pp. 1816-1830, 2014.

[15] M. Montazer and S. M. Seyedpoor, "A new flexibility based damage index for damage detection of truss structures," Shock and Vibration, vol. 2014, Article ID 460692, 12 pages, 2014.

[16] J. Gao, Q. Yuan, P. Li, Z. Feng, H. Zhang, and Z. Lv, "Effects of bending moments and pretightening forces on the flexural stiffness of contact interfaces in rod-fastened rotors," Journal of Engineering for Gas Turbines and Power, vol. 134, no. 10, Article ID 102503, 2012.

[17] J. Li, Z. Li, H. Zhong, and B. Wu, "Structural damage detection using generalized flexibility matrix and changes in natural frequencies," AIAA Journal, vol. 50, no. 5, pp. 1072-1078, 2012. 
[18] M. R. N. Shirazi, H. Mollamahmoudi, and S. Seyedpoor, "Structural damage identification using an adaptive multi-stage optimization method based on a modified particle swarm algorithm," Journal of Optimization Theory and Applications, vol. 160, no. 3, pp. 1009-1019, 2014.

[19] Q. Yuan, J. Gao, and P. Li, "Nonlinear dynamics of the rodfastened Jeffcott rotor," Journal of Vibration and Acoustics, vol. 136, no. 2, Article ID 021011, 2014. 


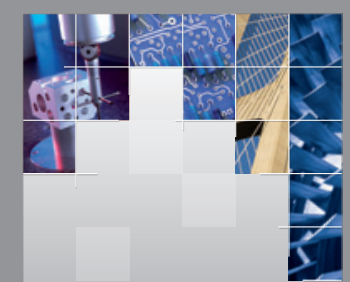

\section{Enfincering}
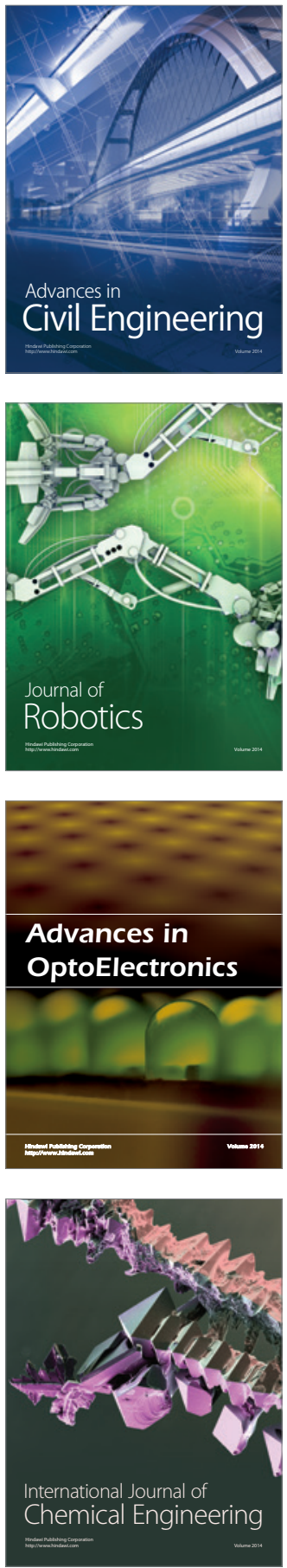

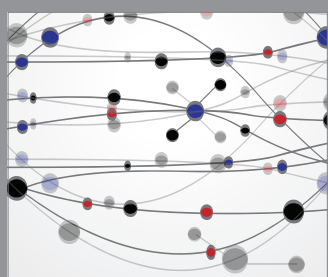

The Scientific World Journal

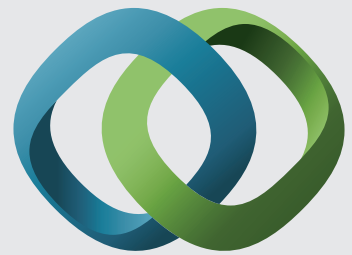

\section{Hindawi}

Submit your manuscripts at

http://www.hindawi.com
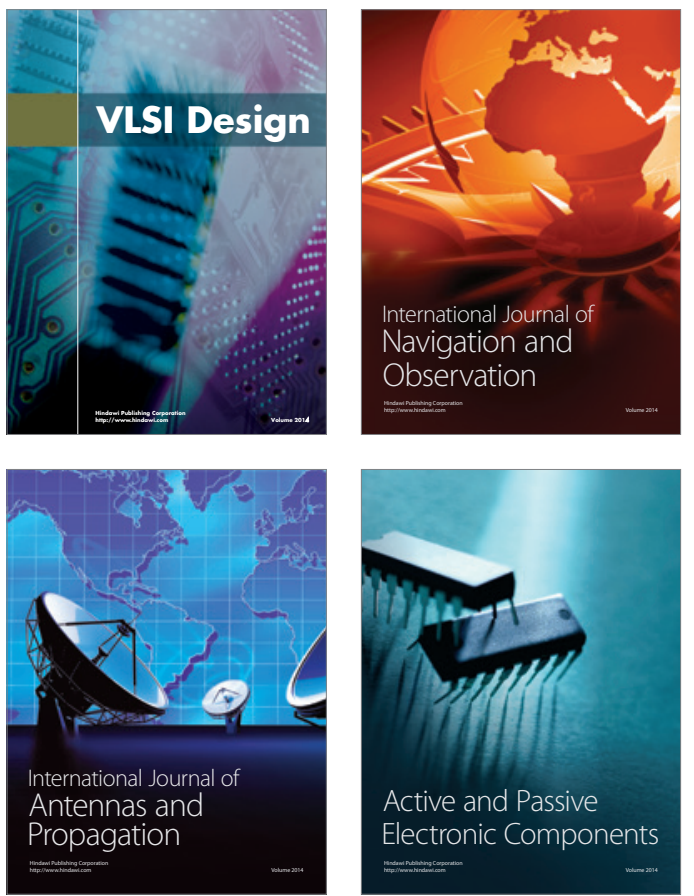
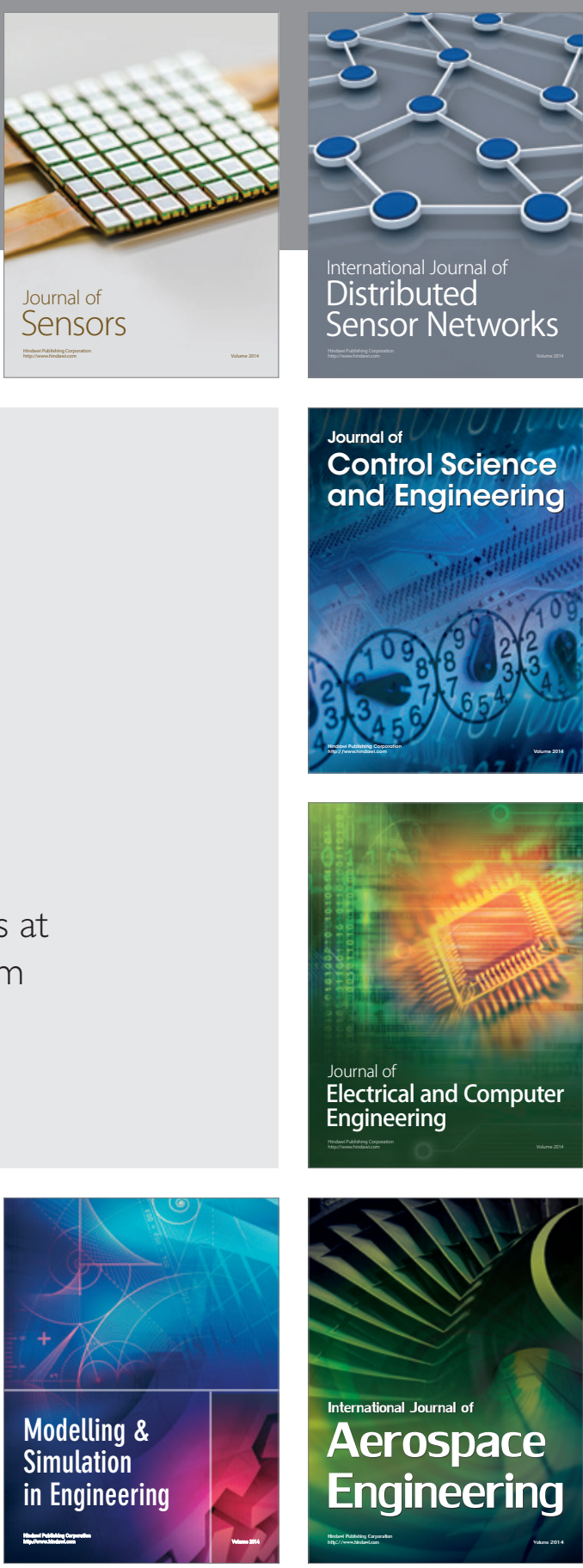

International Journal of

Distributed

Sensor Networks

Journal of

Control Science

and Engineering
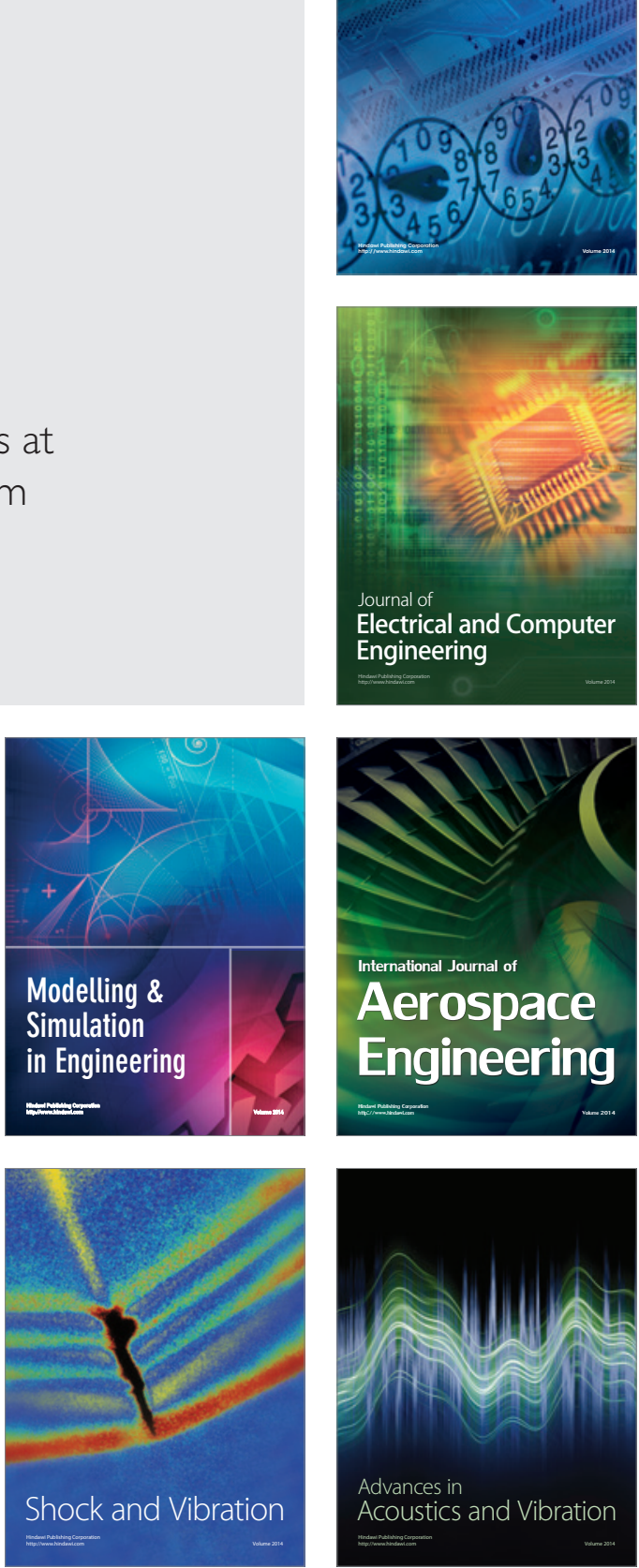